\title{
Comparison of salinity and drought stress effects on abscisic acid metabolites activity of cytokinin oxidase/dehydrogenase and chlorophyll levels in radish audtabacco
}

\author{
Radomira Vankova, Alena Gaudinova, Petre Dobrev, Jiri Malbeck, Daniel Haisel, Vaclav Motyka \\ Institute of Experimental Botany AS CR, Rozvojova 263, 16502 Praha 6, \\ Czech Republic \\ e-mail: vankova@ueb.cas.cz
}

\begin{abstract}
Summary. Stronger negative impact of salinity (100 mM NaCl for 4 days) on radish plants in comparison with tobacco ones, correlated with more profound decrease of bioactive cytokinins and higher elevation of activity of the main cytokinin degrading enzyme cytokinin oxidase/dehydrogenase and abscisic acid in the former species.
\end{abstract}

Key words: abscisic acid, cytokinin, radish, salinity, tobacco.

\section{Introduction}

Plant hormones play an important role in plant response to abiotic stresses, by both stimulation of defence pathways and regulation of plant growth and development. The key hormone in the response to stresses associated with dehydration is abscisic acid (ABA). Its levels were followed during the response to salinity or drought in two species differing in their stress tolerance - radish and tobacco. As cytokinins $(\mathrm{CKs})$ play an important role in regulation of growth and delay of senescence, their level was determined as well.

Regulation of plant hormone (ABA and $\mathrm{CK}$ ) pool during the response to salinity or drought stress.

\section{Material and methods}

Radish (Raphanus sativus L. cv. Rampouch) and tobacco (Nicotiana tabacum L. Samsun) plants were cultivated in soil in a growth chamber (SANYO MLR $350 \mathrm{H}$ ) at $16 / 8 \mathrm{~h}$ light/dark regime, $130 \mu \mathrm{mol} \cdot \mathrm{m}^{-2} \cdot \mathrm{s}^{-1}$, at $23^{\circ} \mathrm{C}$. Plants at the stage of 4 weeks (radish) and 5 weeks (tobacco) were subjected to salinity stress $(100 \mathrm{mM} \mathrm{NaCl})$ or drought stress (withdrawal of watering) for 4 days.

Phytohormones were determined in leaves of control and stressed plants according to Dobrev and Kaminek (2002). ABA levels were quantified using two-dimensional HPLC according to Dobrev et al. (2005). Content of $24 \mathrm{CK}$ metabolites was estimated by LC/MS (Dobrev et al. 2002). The cytokinin oxidase/dehydrogenase (CKX) activity was determined by a modified radioisotope method described by Motyka et al. (2003). Chlorophyll a and b, $\beta$-carotene and xanthophyll cycle pigments were analysed by HPLC.

\section{Results and discussion}

Salinity stress imposed significantly milder effect on tobacco plants than on the radish ones, which became wilty. Tobacco was also less affected by the four-day drought (RWC decrease by ca $11 \%$ ), while radish, as a spring species, was more severely stressed (RWC decrease by ca 19\%). In radish, chlorophyll a level exhibited mild decrease 
(by ca 10\%) in salinity and more profound in drought (by ca $20 \%$ ). Chlorophyll b content was significantly lower, but followed the same trend. In tobacco, salinity caused stronger suppression of chlorophyll a levels than drought. The amount of $\beta$-carotene and xanthophyll cycle pigments followed similar trends as chlorophyll a in both species.

Higher sensitivity of radish to applied abiotic stresses was also reflected by the changes in the ABA content. Salinity and drought caused in radish 2.9 and 4.7 - fold ABA increase, respectively. In tobacco, the effect of salinity was very mild (1.2 fold increase), while drought imposed $2.4-$ fold ABA increase. The basal level of ABA was, however, much higher in tobacco, so in spite of relatively lower elevation, ABA content in stressed tobacco plants was more than three times higher than in radish.

Salinity imposed in radish leaves significant decrease in the level of bioactive CKs (by ca $25 \%$ ), the effect of drought was much stronger (bioactive CK decrease by ca $73 \%$ ). Similar trend was observed in case of CK phosphates, the immediate biosynthetic CK precursors. CK deactivation products, CK N-glucosides, negatively correlated with bioactive CKs. Mild, but significant, increase was observed in salt stress, while profound elevation (by ca $100 \%$ ) was found in drought. The level of reversible glucoconjugates, CK O-glucosides, was relatively low, being higher in salinity than in drought. The same trend was observed in case of cis-zeatin derivatives.

Higher drought tolerance of tobacco plants correlated with a milder decrease of bioactive CKs under this stress conditions than in salinity. In case of CK phosphates, significant reduction was observed in both stresses. No significant change was found in $\mathrm{CK} \mathrm{N}$-glucosides. CK O-glucosides elevated under salt stress, but decreased in drought, which might indicate that CK deactivation was suppressed under the latter conditions.

When the activity of the main CK degrading enzyme, CKX, was monitored, higher elevation was found in drought than in salinity in radish. Response to both stresses was in tobacco associated with mild CKX decrease.
The achieved results indicate that higher stress sensitivity of radish was associated with more profound decrease of bioactive CKs, accompanied with stimulation of their degradation. More drought tolerant species, tobacco, exhibited also decrease in bioactive CKs, however, relatively low CK degradation by CKX as well as low CK deactivation by $\mathrm{CK} \mathrm{O}$-glucosylation seems to indicate that mild stresses are associated with tendency to maintain the level of bioactive CKs, at least during early stress period. Both $\mathrm{ABA}$ and CKs seem to play an important role in abiotic stress response, being tightly regulated in dependence on stress strength.

\section{Acknowledgements}

The work was supported by MEYS project no. LD11073.

\section{References}

Dobrev P. I. \& Kaminek M., 2002, Fast and efficient separation of cytokinins from auxin and abscisic acid and their purification using mixed-mode solid-phase extraction, J. Chrom. A. 950: 21-9.

Dobrev P. I., Motyka V., Gaudinova A., Malbeck J., Travnickova A., Kaminek M. \& Vankova R., 2002, Transient accumulation of cis- and trans-zeatin type cytokinins and its relation to cytokinin oxidase activity during cell cycle of synchronized tobacco BY-2 cells, Plant Physiol. Bioch. 40: 333-337.

Dobrev P. I., Havlicek L., Vagner M., Malbeck J. \& Kaminek M., 2005, Purification and determination of plant hormones auxin and abscisic acid using solid phase extraction and two-dimensional high performance liquid chromatography. J. Chrom. A. 1075: 159-66.

Motyka V., Vankova R., Capkova V., Petrasek J., Kaminek M. \& Schmülling T., 2003, Cytokinin-induced up-regulation of cytokinin oxidase activity in tobacco includes changes in enzyme glycosylation and secretion, Physiol. Plant. 117: 11-21. 